\title{
Gestión del agua en estancias ovejeras según algunos manuales de finales del siglo XIX
}

\author{
Water management at sheep rearing \\ ranches according to three late 19th century handbooks. \\ Pablo Ernesto Suárez \\ Universidad Nacional de Rosario-Argentina \\ pablosuarezdg@gmail.com
}

\section{Resumen:}

El agua fue un factor fundamental en la organización y en la producción de las estancias ovejeras, aunque no haya merecido demasiados estudios específicos por parte de los historiadores. El presente trabajo se propone analizar qué importancia le asignaban al agua tres autores considerados claves para el período: José Hernández, Estanislao Zeballos, Carlos Lemée y Godofredo Daireaux. Los autores proponen ciertas intervenciones para poner el recurso a disposición de la producción; para ello, se requerían ciertas inversiones en infraestructura y conocimientos que combinaron saberes antiguos con tecnologías de avanzada para la época.

Palabras clave: agua, estancias, ovejas, lana, tecnología.

\begin{abstract}
Water was a key factor for organisation and production at sheep rearing estancias (ranches). Even though it attracted little attention by historians. The present work intends to survey how relevant water was for three authors who were deemed as essential for that period: Hernández, Lemée, Zeballos and Daireaux.They propose a number of water sourcing techniques and devices for the purpose of sheep farming, which required some investment in infrastructure and a combination of traditional know-how and state of the art technology.
\end{abstract}

Key words: water, estancias, sheep, wool, technology. 
El agua vincula y relaciona. No solo une en su fluir a la materialidad del mundo; en el plano de la escritura histórica el agua logra poner en contacto realidades que han sido abordadas en forma separada. Esto genera un gran desafío para quienes pretendan escribir sobre este tema. Al tener que atender varias referencias, se corre el riesgo de incurrir en análisis parciales o superficiales de algunos temas relacionados. La economía, las representaciones, las políticas territoriales, el ambiente, la ecología son terrenos por los que el agua circula, deteniéndose en algunos, apurándose en otros, abarcando en su complejidad una diversidad de temas y enfoques, que la tarea de los historiadores integra en trabajos parciales, que facilitan futuras síntesis integradoras.

Reconociendo esas limitaciones, este artículo se enfocará en un tema poco abordado por la rica historia agraria argentina, un área de estudios que captó grandes (en cantidad y calidad) esfuerzos historiográficos, debido quizás a las características espectaculares del crecimiento del sector agropecuario en el siglo XIX. (Sesto 2005; Gelman y Barsky, 2001; Sábato, 1989; Gallo, 1983). Si bien la dimensión técnica también orientó esfuerzos (Sesto, 2005) la cuestión de la gestión del agua en general y en las estancias en particular, no ha recibido demasiado tratamiento.

Se analizará (a partir de algunas publicaciones que consideramos significativas) la importancia que la dotación y el manejo hídrico tuvo en las estancias ovejeras en la visión de cuatro autores del agro bonaerense a fines del siglo XIX. La elección de los textos obedece por un lado a la importancia de esos autores en la historia de la literatura argentina, a que todos tuvieron a su cargo explotaciones ovejeras y finalmente a que dejaron testimonio de su experiencia y su visión del problema con un fin práctico que promovía el conocimiento del tema y al mejoramiento de las empresas orientadas a esa actividad.

El acento estará puesto en su visión de la importancia del agua, su disponibilidad y su manejo, las intervenciones que requirió y la valoración subjetiva -por parte de los dueños de las estancias- en ese contexto histórico.

Cabe señalar que el formato "manual de instrucciones" no era una novedad en esos años. Hay-por lo menos- dos antecedentes muy conocidos en la región, algunos de los cuales están citados en los que hoy analizamos; nos referimos a los textos de Pérez Mendoza (1863) y Harrat (1885). Estos trabajos encuentran en España un antecedente firme en Nicolás Casas, un prolífico veterinario español que venía publicando piezas de este tipo desde la década de 1830.

Los textos que reseñaremos fueron escritos en la década de 1880 por cuatro autores cuya producción y participación en los debates del momento, hacen pertinente su tratamiento en conjunto. En el caso de José Hernández (nacido en 1834), la obra que nos convoca es de 1881 y es dos años posterior a la Lavuelta de Martín Fierro, la segunda parte de su icónica obra, uno de los libros más importantes de la literatura argentina. Fue un periodista prolífico y activo participante de los debates políticos rioplatenses en el último tercio del siglo XIX. Fue diputado y senador provincial en la provincia de Buenos Aires. 
También consideraremos los textos de Daireaux (1887) y Carlos Lemée (1882), ambos franceses radicados en el país. El primero, nació en 1859 y arribó a la Argentina en 1868 para dedicarse al comercio. Luego se convertiría en estanciero y a partir de entonces será una referencia de múltiples actividades: periodista, escritor, funcionario en el ámbito educativo y un importante promotor de la cultura en general. Volcará en sus numerosos libros su experiencia en el campo argentino, alternando relatos de ficción con manuales como el que hoy comentamos. Lemée, nacido en 1828 y llegado a estas tierras en 1855, al igual que su paisano antemencionado, fue un activo protagonista en los intercambios sobre temas agropecuarios en el país, con una gran cantidad de textos relacionados a la temática, alternando la ficción con escritos técnicos. De este último carácter, exhumaremos su Tratado (1882).

Finalmente, el texto de Estanislao Zeballos (1888), es parte de su trilogía propagandística de la Argentina. Se trata de uno de los intelectuales argentinos más destacados del período con una amplísima foja de servicios en la función pública, donde ocupó cargos desde diputado provincial hasta ministro de relaciones exteriores en tres ocasiones. Zeballos tuvo múltiples áreas de interés intelectual: desde las ciencias naturales al derecho, geología, geografía, historia, etnografía habiendo realizado aportes destacados en varias de esas disciplinas

Si bien las opiniones de los autores deben confrontarse con los documentos de las explotaciones reales, planillas, inventarios y todo tipo de registro, importa considerar los criterios que regían en esas estancias, y develar algunas claves acerca de la circulación de saberes y criterios técnicos de la época. El cruce con esos otros registros dará cuenta del grado de la eficacia e incidencia reales de estas proyecciones en la gestión de las empresas. Sigue una breve contextualización respecto del mundo de la lana en el período, para luego sí pasar a analizar los textos elegidos como referencia principal.

\section{Los estudios sobre la producción lanar en el siglo XIX.}

La importancia económica de la cría de ovejas en la región sur Río de la Plata fue insoslayable, como queda claro en los trabajos de la renovación historiográfica de fines de siglo pasado (Sábato, 1989; Amaral, 1998).

Ya desde la época colonial, se sabe de la existencia de pequeñas majadas, e incluso de algunas ínfimas exportaciones de lana. Investigaciones realizadas con inventarios post mortem, mostraron que el ganado ovino fue parte importante de los patrimonios de las estancias a partir de 1820 (Garavaglia, 1998; Amaral, 1998). Aunque no aportó significativamente al consumo doméstico ni a las exportaciones de la región, ambos dominados abrumadoramente por los productos de origen bovino, sí existió un mercado interno para el vellón sobre todo en las provincias del litoral. Durante la segunda década del siglo hubo algunos intentos de mestizaje, mediante la importación de ovejas de raza, para mejorar las lanas locales, pero esos ensayos nunca lograron (por diversas causas) generar majadas consistentemente puras. (Zeballos, 1888; Barsky y Djenderedjian, 2003: 175 y ss). 
En la década del 40, las estancias ovejeras que asombraron a los viajeros sentarían las bases sobre la cual crecerían las exportaciones de lana y de cueros ovinos, para lo que fueron necesarias algunas modificaciones respecto de las técnicas anteriores.

Las lanas enviadas a los mercados internacionales hasta la década de 1840 fueron ordinarias, por las variedades ovinas que se criaban en el Plata. Sin embargo, el aumento en los valores alentó la mejora en las razas y la introducción de novedades tecnológicas como aquellas vinculadas al manejo del agua, la construcción de viviendas para el personal, galpones para la esquila en verano y graserías para aprovechar el sebo. (Rayes 2014: 4)

Los buenos precios internacionales, lo convertirían en un fuerte atractor de inversiones, un gran impulso de la expansión de la frontera productiva y un dinamizador del mercado de tierras. El ganado vacuno fue la punta de lanza de aquella expansión; preparando las tierras para la posterior llegada de las ovejas (Sábato, 1989: 38); mientras las estancias ovejeras se consolidaban en los lugares preferenciales, las tierras más cercanas a los puertos de exportación (Gelman y Barsky, 2001).

El ciclo caracterizado por Sábato como "la fiebre del lanar" duraría hasta 1870. Se trataría de una prosperidad inestable, debido a un escenario de baja de precios causada por una sumatoria de sucesos internacionales, entre los cuales se destacaron las medidas proteccionistas de Estados Unidos y la guerra del Paraguay.
No obstante, y con el proceso de expansión de la agricultura extensiva en secano en vías de consolidación, todavía en 1890 la lana constituía el $35,5 \%$ de las exportaciones, sobre un 65,5 del total compuesto por exportaciones de origen ganadero (Cortés Conde, 1998: 68).

La aparición de tecnologías que permitieron comercializar carnes primero congeladas y luego enfriadas modificó los parámetros de producción ovina, generando un nuevo mestizaje que priorizó el volumen cárnico, en el llamado proceso de “desmerinización” que abrió paso a la raza Lincoln, con mejor rendimiento para fines alimenticios. Una vez que el frigorífico encontró la forma de enfriar animales de gran volumen, las ovejas cederían su liderazgo en las tablas de exportación y también entregarían en su derrota las mejores tierras a los campos mixtos destinados ahora a la rotación agrícola-ganadera. La producción ovina perduraría, pero ya desplazada a zonas marginales (Frid, 2007; Sábato 1989).

Consideramos importante destacar la particular configuración que revistió la mano de obra en estos establecimientos. Aunque se reseña la existencia de grandes estancias con personal asalariado, las explotaciones ovejeras permitían la posibilidad de desarrollo (en su interior) de formatos más pequeños, con microempresas que eran llevadas adelante por grupos familiares de puesteros o mayordomos (Gelman y Barsky, 2003: 123). Por su parte, Colombo y Balsa, (2007), muestran para el caso puntual de Saladillo, la convivencia de una gran variedad de dimensiones en las explotaciones, con distintos formatos de posesión de la tierra y gestión de la producción. 
Esos inmigrantes eran muy valorados por los estancieros, y es por eso, por lo que generalmente ocupaban el cargo de Administradores o Mayordomos, jerárquicamente por encima de los peones comunes y con mayores responsabilidades y dividendos. En los años de caída en el precio de la tierra, (que a veces coincidían con los de altos salarios) estos trabajadores podían acceder a la propiedad del suelo, generándose así una clase de pequeños productores ovinos que tendría un peso importante en el volumen total producido.

Las grandes estancias ovejeras requerían mano de obra estable (y más capacitada) que la estancia bovina, además de requerir una gran cantidad de trabajadores para el momento de la esquila (Posada y Iuliano, 1995). Hilda Sábato (1989) refiere una gran transformación en la vida de las estancias durante la temporada de esquila (octubre-enero). Hasta aquí un panorama general respecto de la producción ovina.

\section{Aportes y perspectivas en relación con el uso del agua en el agro pampeano}

En Argentina, los estudios sobre el agua tienen un desarrollo desigual, con especial fortaleza en las provincias de Mendoza, La Pampa y Córdoba (Larsimont y Grosso, 2014; Langhoff, M. L., Geraldi, A., Rosell, P. 2017) con referentes como Gabriel Garnero (2014a, 2014b) para esta última provincia, con una importante serie de trabajos de carácter histórico. Como vemos, se trata más bien de regiones alejadas de la producción en secano. Los aportes de Guillermo Banzato (2014, 2015, 2016), abordan conflictos por el agua en el territorio de la provincia de Buenos Aires, con trabajos que analizan las políticas promovidas desde los estados provincial y nacional con respecto al tema de las inundaciones, problemas que -como veremos en las reseñas de Zeballos y Hernández-, estaban en la agenda y en los presupuestos de los gobiernos provinciales ya desde la década de 1870.

Sobresalen también las investigaciones de Andrea Reguera sobre la administración de las estancias pampeanas (1999a, 1999b, 2000) donde documenta la preocupación de los puesteros y mayordomos respecto del agua; aunque prioriza el enfoque desde el "riesgo", jerarquiza ese punto de la gestión del recurso, y pone el foco en inundaciones, temporales, sequías, etc. Podemos suponer que los temas y modos que preocuparon a Ramón Santamarina y sus empleados, eran preocupaciones comunes a todos los productores de la región.

Roberto Schmit (2015) nos brinda un panorama sobre las estancias agropecuarias en la provincia de Entre Ríos. Más allá de sus particularidades, la evolución productiva de estos establecimientos durante el siglo XIX ofrece grandes similitudes con los casos bonaerenses. Una nueva luz respecto del uso del agua podrá surgir de los trabajos arqueológicos como los que se han realizado sobre algunas estancias de la región sur del Salado, como los trabajos de Bagaloni (2018) y Facundo Gómez Romero (2016); este último mencionando los hallazgos realizados en pozos y aljibes que funcionaron como repositorios de basura.

También desde la arqueología, y complementando con un corpus similar al que hoy abordamos, se hallan los trabajos de Fernando Brittez, aunque su indagación apuntó más a dar cuenta de la 
cultura material y de la organización espacial de la estancia, con referencias a la teoría del panóptico desarrollada por Foucault (Brittez, 2006). Creemos que incorporar la problemática del agua hará que esos mismos documentos den nuevas explicaciones a las configuraciones territoriales resultantes.

\section{El agua en la estancia: un análisis a través de los manuales de época}

En una estancia ganadera el agua tiene diversos usos: el consumo cotidiano de quienes viven y trabajan, el de los propios animales, el riego de las plantas forrajeras y también la higiene de las reses. Tiene también distintos puntos de obtención: lluvia, pozos y aguadas naturales.

La pasividad a la que fue reducida el agua en los relatos históricos nos la presenta como un "factor de producción" ante el cual el empresario se posiciona como un mero "aprovechador" que emplea el recurso "dado" por la naturaleza, que fungía, eso sí, como valorizador de las tierras (Amaral, 1998: 65). En realidad, el proceso consiste más bien en una apropiación del recurso mediante maquinarias, herramientas y otros dispositivos de infraestructura para incorporarlo a la producción. En este sentido los autores reseñados juzgarán con severidad los errores en la gestión de las estancias y propondrán sus soluciones a partir de su experiencia y de los avances tecnológicos a los cuales están atentos.

¿Por qué y para qué se hicieron estos manuales? Salvo el caso de Zeballos que no es un manual, sino más bien una descripción propagandística de los progresos que el país había realizado (visto en el contexto de su trilogía) los otros textos tienen una función claramente técnica.

A apenas tres años del lanzamiento de la primera, Casavalle dice en la Advertencia que encabeza la tercera edición, que hace treinta años "La ganaderia representaba lo contrario de una industria" (II) y se congratula de la evolución producida. Señala que los manuales europeos no dan cuenta de las condiciones de producción locales y que el libro de Hernández ha venido a completar un vacío y "favorecer por el conocimiento de las doctrinas y principios que desenvuelve, á la principal industria de la Nación." (II) Por eso este libro "mas se halla destinado á instruir que á deleitar; mas á servir los intereses positivos, que al mero entretenimiento." (I).

Por su parte, Daireaux, comenta en 1908 en el prólogo a la cuarta edición (la primera es de 1887) que los preceptos que se dan en el libro estaban "dirigidos todos a acelerar en lo posible la trasformación del rudo desierto pampeano en praderas feraces" (5) y expresa su alborozo por ver que el estanciero moderno ya no es un simple criador de hacienda, dicho con el cual justifica su interés en la estancia mixta, que incluya la agricultura como una actividad protagónica. Del mismo modo que Casavalle, defiende la experiencia situada de los productores argentinos y la necesidad de este tipo de publicaciones, ya que "en los libros europeos, por buenos que sean, el criador argentino no puede encontrar resueltas, ni siquiera debatidas, mil cuestiones sumamente interesantes para él [...]" (11) 
Algunos de los autores explicitan la intencionalidad de su trabajo. José Hernández, en una velada crítica a la intelectualidad del momento será terminante

En nuestro país se escribe muy poco sobre industria rural.

Todos cuantos se dedican al cultivo de la inteligencia y al comercio de las letras, dan á sus fuerzas direcciones estrañas á la industria Nacional, y recorren el vasto campo de la literatura, cultivándola en la forma galana de la poesía, en las originales invenciones del romance, en los ardientes arrebatos de la prosa política, en las delicadas investigaciones históricas, en las disertaciones doctrinarias del derecho, ó persiguiendo otros variados propósitos, de muy elevado interés público, peroágenos á las exigencias industriales y á la naturaleza de nuestra riqueza fundamental.

Nuestro libro, por su carácter y objetos, será sin duda una escepcion al giro intelectual de la época [...] (3-4).

Las sucesivas y cercanas ediciones de los libros dan cuenta de cierta avidez del público que evidentemente corresponde al interés de los autores, que buscaban argumentar en un lenguaje sencillo para intentar llegar a una audiencia amplia.

[...] escribimos [...] para el gran número de los que, teniendo estancia, sin ser estancieros, necesitan siquiera saber algo, teóricamente, del oficio, para poder dar una dirección juiciosa al manejo de sus negocios de campo; de los principiantes que quieren formar estancia, ó en escala menor, ocuparse de cria de ganado; de los extranjeros que, cada vez en mayor número, vienen aquí á dedicar su trabajo y sus capitales al negocio de estancia; de todos aquellos, en fin, que, por algún motivo, deseen ó necesiten poseer algunos datos ó completar los que ya tengan sobre la ganadería en la República Argentina (9).

\section{El agua de lluvia}

Antes que nada, tengamos en cuenta lo siguiente: en términos generales, una oveja consume aproximadamente cuatro litros de agua por día. De modo que para satisfacer el consumo de los animales de una estancia era necesario poner una gran cantidad de agua a disposición de las majadas. Daireaux dice que "teniendo pasto verde, la oveja casi no pide agua, o por lo menos se contenta con muy poca" (174), planteando incluso que se puede dar agua cada dos días, pero tanto él como Lemée plantean que, en el verano, debido a la forma en que los animales sufren el calor, la hidratación debe ser necesariamente diaria y en horas frescas, "a la madrugada o a la oración" (66). Aspecto que también destaca Hernández (296) cuando dice que, si bien beben poca agua en general, en los meses de verano debe reforzarse la hidratación.

La imposibilidad de manejar la lluvia no implicó que los estancieros se quedaran pasivamente a administrar el régimen pluvial; de modo que la elección de los campos se convirtió en un aspecto clave de la empresa. Las lluvias son fundamentales para cargar las lagunas, los arroyos y todos los reservorios de agua dispuestos para su contención. Un buen régimen es óptimo, además, porque 
prácticamente no demanda inversiones. Por eso Daireaux (159) va a valorar como "una gran suerte" la caída de aguaceros que llenen las lagunas, para evitar cansar la majada en la búsqueda de aguadas y Hernández va a ironizar acerca de los estancieros del norte, "que pasan la vida entera pidiendo agua” (Hernández, 1884: 68).

Aunque Carmen Sesto (2005: 107) refiere la existencia en alguna estancia de estructuras de riego subterráneo, en los textos reseñados, las lluvias eran la mejor garantía para el crecimiento de las pasturas en los campos.

Esa dependencia ubica a las empresas ante la cláusula secreta de todos los productores agropecuarios: implorar por un lado las lluvias... e inmediatamente implorar por su justa medida, ya que existen enfermedades de las ovejas que encuentran un medio favorable en los ambientes de excesiva humedad.

Dice Zeballos "No se trataba sino de una epidemia del tipo enteritis aguda producida por el pasto, desnaturalizado á consecuencia de la abundancia y frecuencia de las lluvias é inundaciones de 1884. (283) o cuando se refiere al Enteque o Calcinosis Enzoótica, afirma "Esta enfermedad [...] proviene del modo forzosamente primitivo que tenemos aquí y tendremos todavía por mucho tiempo, de criar las haciendas a puro pastoreo y en muchas partes, en campos bajos y anegadizos[1] (285). Y cita a continuación a Birabén quien propone inversiones en infraestructura, con intervenciones en el paisaje del propio establecimiento: secar los bañados y canalizar los arroyos para impedir las inundaciones y de esa forma evitar el sagüaipé [2] (285).

Pero "la solución no está muy lejos del mal" como decía Daireaux (313), pues resulta que la hoja del sauce era un buen antídoto para el sagüaipé y por eso se aprovechaban las orillas de los mismos arroyos para que las ovejas lo comieran y se previniese así la enfermedad. Como vemos, el espacio no era concebido como un ente ante el cual posicionarse pasivamente, aceptando sus mandatos y limitaciones, sino que era intervenido en función del interés empresarial para maximizar su rendimiento. Cuando el paisaje original no era suficiente solución, también Daireaux tenía muy clara la importancia de las soluciones estructurales sabiendo que "La canalización de los campos bajos será probablemente el mejor remedio para el enteque y la lombriz..." (469).

Esta mirada combinada y amplia que articulaba paisaje e intervenciones, lo hizo postular que, para elegir un buen campo, debía priorizarse la buena tierra, ya que "el agua se encuentra en todas partes y será siempre menos costoso buscarla que tener que remover algún día la estancia por no convenir la situación primitiva..." (31).

La ventaja de los terrenos bajos para retener el agua se perdía en las épocas lluviosas porque como vimos la inundación afectaba a los animales de diversas maneras, incluso con riesgos de ahogamiento cuando las lluvias eran intensas; por eso eran especialmente valorados los campos que tuvieran lomadas donde poder llevar las majadas en caso de lluvias abundantes. 
[...] a pesar de las ventajas que presenta el terreno alto, debe tener sus partes bajas para resistir durante las sequías, diremos que el tipo mejor de un terreno de estancia es el compuesto de albardones altos y anchos, cortados de cañaditas. (21)

Mientras que Zeballos (1888: 240) propone analizar en cada campo el estado de los pastos y el régimen de lluvias, para ver cuántas ovejas puede alimentar, Daireaux propuso una fórmula para saber cuánta hacienda podía soportar un campo: "Se puede tomar por base el máximum de hacienda que puedan sostener las partes bajas del campo, en caso de sequía, y las partes altas, en caso de inundación. De ese modo, por lo menos se salvarán los intereses" (23).

Ya vimos que el exceso de aguas era un problema recurrente. Los manuales proponían estrategias específicas como la canalización de las zonas inundadas o el desplazamiento de las majadas a las zonas altas, aunque sin soslayar las obras de defensa y soluciones integrales y definitivas para las distintas zonas. El pensamiento estratégico de Zeballos le permitió ver un poco más allá de los problemas locales de los productores.

En su diálogo con Ameghino respecto de las inundaciones en la Provincia de Buenos Aires (un texto clave, del cual preferiremos no abusar) plantea lo siguiente:

Los campos argentinos han modificado fundamentalmente su aspecto salvaje, y la acción privada ha construido canales de desagüe en unas partes y escavado depósitos permanentes de agua en las otras, anticipándose así, á las medidas oficiales que un día será necesario adoptar para regularizar la distribución de las corrientes, evitando que inunden una región, mientras queda sedienta otra comarca inmediata. (253)

Ambos eran conscientes de que esas intervenciones debían promoverse desde el Estado. Más allá de los deseos de Hernández, (1884: 11) que los veía como una doble solución (a los anegamientos y al problema del transporte) la imposibilidad de convertir los pequeños cauces de la provincia de Buenos Aires en canales navegables (básicamente por sus pequeños caudales en la temporada seca) vino a ser subsanada por el tendido ferroviario, que desde muy temprano se convirtió en el medio más eficiente para que las lanas llegaran a su destino porteño, en un servicio que era más eficiente y además económicamente más rentable. (Sábato, 1989: 231).

En el otro extremo de las inundaciones se encuentra la sequía. La mirada de largo plazo de los estancieros los obliga a estar muy pendientes de las soluciones de contingencia, para no sufrir el desastre que ocasiona la carencia de agua. Zeballos menciona una estancia en Cañuelas donde la ausencia de agua implicó la pérdida de 30.000 animales (250).

Y Daireaux se muestra terminante al respecto. Aconseja mantener siempre listos los jagüeles, 
[...] pero aconsejamos á todos los amigos del progreso de reemplazarlo cuanto antes por algunos de los tantos sistemas nuevos, á cual mejor, que cada día salen á luz, [...] No hay en esto que invocar pretexto de economía: economicen lo que puedan y en lo que puedan; pero no sobre los medios de proporcionar agua abundante a las haciendas [3]. (396)

Los fenómenos climáticos asociados con el agua presentan muchas veces situaciones límite que activan o redimensionan a otros factores productivos sobre los cuales se ha tenido una mirada demasiado unidimensional. La sequía, por ejemplo, ponía de relieve la gran utilidad de los alambrados, que evitaban la mezcla de majadas, una situación no deseada por los cabañeros que pretendían "diseñar" sus planteles manteniendo un estricto control sobre los cruces entre animales de distinta raza:

Una de las causas más frecuentes de mixtura es la escasez de agua. Cuando por una seca prolongada se van secando las últimas lagunas, las ovejas vienen de lejos disparando para el agua y a menudo sucede que en la aguada se encuentra otra majada bebiendo y allá se mixturan. (Daireaux, 1904: 175)

A esta defensa del alambrado se sumará Lemée, quien agregará razones de índole sanitarias, ya que el cercado de los lotes impedirá el contagio de los planteles sanos, con algun animal portador de sarna a la que identifica como "la primera mejora que reclama nuestro ganado lanar" (61).

\section{El agua de los pozos}

La extracción de agua subterránea fue una solución para resolver el problema del abastecimiento en regiones sin cauces superficiales o con un régimen de lluvia mezquino. Hasta la aparición de los molinos (Sbarra, 1973) esta práctica fue muy común desde la época colonial en varias regiones del actual territorio argentino, con pequeñas modificaciones en el diseño de los pozos y modelos de aljibe y jagüeles.

Veamos como pinta Hernández (1884) el cuadro de pasado de la producción ganadera:

Ya se pasaron los tiempos en que el poblador de una Estancia á cuarenta ó cincuenta leguas de Buenos Aires, se situaba con su hacienda y su tropilla de caballos en la costa de un arroyo, empezaba por hacer un toldo con dos cueros, y allí se refugiaba mientras buscaba algunos palos, cortaba la paja, armaba su rancho, le ponía un quincho embarrado, y así construía una habitación en la que vivía años enteros, sin mas puerta que un cuero atado con unas guasquillas. (109)

En el revés de la trama de su crítica al estancamiento y la falta de inversión como conducta repetida en los estancieros, Hernández se propuso plantear qué tipo de estancia debía concretarse para lograr los desafíos que la demanda internacional propuso a los hacendados argentinos: volúmenes de producción y niveles de calidad que serían inalcanzables con las viejas estructuras técnicas y conceptuales. Dealgún modo, esta proliferación de manuales en sincronía nos sugiere que estábamos 
en un contexto de diagnósticos negativos acerca de los modos en que se gestionaban las estancias.

Y en esas inversiones, el agua es primordial: "El primer trabajo que se debe hacer, es de cavar un pozo." dice Daireaux (1904: 37). "Es preciso tratar de que se haga en un sitio conveniente y cómodo para los usos ulteriores de la casa. Si el agua sale salobre, ... es bueno buscar en otro lugar agua potable" (37).

Los pozos surgentes son aquellos en el agua asciende a la superficie por una diferencia de presión atmosférica, mientras que en los semi-surgentes se requiere algún medio mecánico para completar su ascensión.[4] Pero "pozo" se le llama también al dispositivo por medio del cual se extrae el agua y los había de dos tipos: el pozo de balde y el pozo de manga.

Para uso doméstico o consumo humano, los autores proponen "pozo de balde", no muy distinto a un aljibe, que es el modelo que se coloca cerca de las habitaciones. El llamado "balde" es una pieza de cuero de forma semi-esférica que podría llegar a cargar varios litros de agua (amén de que existieran de distintos tamaños, de acuerdo con las necesidades de cada uso).

Hernández (1884: 110 -111), por ejemplo, propondrá que en cada puesto se haga un pozo, tanto en la casa del mayordomo como en la del capataz y peones. Aunque con los mismos principios mecánicos, el pozo de manga tenía otras dimensiones y por lo tanto otros usos ya que en la medida en que permitían extraer grandes volúmenes de agua, se ubicaban en las áreas destinadas a circulación o asentamiento de las ovejas y con la fuerza motriz a cargo de animales (bueyes, mulas o caballos), con una notable dilapidación de energía animal, al decir de Zeballos "con una combinación de cuerdas cae al pozo, se llena, sube doblado y derrama en las bebidas dos terceras partes de su capacidad total" (Zeballos,1888: 310)[5].

Hemos definido hasta aquí entonces, los dos formatos de pozo diferenciados solamente por la herramienta contenedora del agua (el balde y la manga), que sube mediante una cuerda con tracción a sangre animal o a pulso humano (dependiendo de las dimensiones y volúmenes requeridos). Pero extraer el agua es apenas el comienzo del proceso. Para que el ganado pudiera beberla, debían disponerse los implementos que lo hicieran posible: las tradicionales canaletas de madera o hierro llamadas "bebidas" y el formato más utilizado, el Jagüel:

El Jagüel es un pozo grande para dar agua a las haciendas; generalmente se cava mas largo que ancho y se usa en él una manga de madera, de hierro o de lona o de cuerpo para sacar el agua. El agua cae en una represa y de allí en las bebidas de madera, de hierro o de material donde la toman los animales. (Daireaux, 1904: 78)

Zeballos sabe diferenciar el sistema tradicional, del que es crítico:

En general se abre un gran pozo, de cuatro varas de boca y la profundidad necesaria: es un jagüel. Algunos lo cavan al borde de una lagunita temporal y la llenan. Es el peor 
bebedero para ovejas, pues, se entierran en el barro y beben agua inmunda. (1888:310)

Del innovador, que encontrará Zeballos en la estancia "Los poronguitos", de Claudio Stegmann. Hijo de un comerciante y estanciero alemán muy cercano a Juan Manuel de Rosas, Stegmann realizó su formación profesional en Alemania y llegó a ser un hombre relevante en la política del período: fue senador provincial y Vicegobernador, además de fundador de la Sociedad Rural Argentina (Flores, 2010).

Como podría suponerse la situación aquí es distinta a la de otras estancias:

Los jagüeles no son charcas inmundas, como se ve con frecuencia, donde menos lo imaginaba uno sino pozos abovedados, con boca calzada para recibir la noria ó el balde. El sistema es sin duda costoso; pero es un gasto reproductivo, pues asegura la buena higiene del agua, su temperatura uniforme y permanencia. (115)

Y por su parte Hernández, afirma:

Los sistemas mas nuevos son los dos siguientes: Hacer un jagüel principal bien hondo, y en varias direcciones; alrededor de él construir otros pozos surtidores, dándoles comunicación subterránea á fin de que el agua que brota de ellos vaya á reunirse en el pozo principal, que es donde está la maquinaria para sacar el agua; estando los otros completamente tapados. (1884: 134)
Queda claro, a juzgar por los manuales de época, que, en la década del 1880, el jagüel (limpio o sucio, chico, o grande, con mayor o menor detalle en la construcción, con diversidad de materiales) era el sistema preferido en las estancias. De todos modos, tengamos en cuenta que los textos de Daireaux y Hernández están siendo concebidos como "manuales" que prescriben los pasos a seguir. Aunque abogaron por las nuevas tecnologías, supieron articular ese impulso con las posibilidades reales y las condiciones de producción al alcance de los estancieros.

Además de las razones económicas y funcionales al interior de cada estancia, la correcta provisión de agua era un requisito legal. El Código Rural vigente en estos años estipula la obligatoriedad de abastecer de agua a los establecimientos y propone sanciones económicas para aquellos estancieros cuyos animales "penetraren por falta de agua en campo ajeno que la tenga". Dichas instalaciones y su proporcionalidad respecto de las majadas serían inspeccionadas por los Jueces de Paz de cada partido. Aunque esas disposiciones "no son obligatorias en las grandes secas declaradas [...] y en que vean las autoridades locales que á pesar de todo, es inevitable la dispersion de las haciendas" (Sociedad Rural Argentina, p. 24).

Hacia fin de siglo aquellas rústicas tecnologías heredadas de la colonia comienzan a convivir con algunas máquinas-herramienta más modernas, algunas con muchos componentes metálicos. Estos cambios se aceleran fuertemente a partir de 1880, al calor de la gran transformación tecnológica que la llamada Primera Globalización estaba generando a nivel mundial. En el agro 
argentino, no sólo la ganadería (en particular la pampeana) habría de transformar rápidamente su tecnología de producción, sino también la agricultura extensiva de secano (que llevaría al país a ocupar un lugar destacado en el comercio internacional de granos en el cambio de siglo). Entre esas innovaciones se ubica la noria. Consistía en un enorme engranaje horizontal, movido por un caballo, que transmitía el movimiento a un sin fin de cangilones (receptáculos de agua) que van subiendo y volcando el contenido a un jagüel.

Hasta la aparición del molino (el primero fue presentado por Miguel Lanús en 1881), la noria era la herramienta más avanzada para extraer agua de los pozos semi-surgentes. Si bien encontramos que en 1873 se elogiaba la noria árabe y se detallaba su construcción totalmente en madera (Anales de la Sociedad Rural Argentina, 1873: 275), en el libro de Hernández ya se menciona una "toda de fierro" y de paso como "el aparato más recomendable para abastecer de agua a los establecimientos" (1884: 134).

A diferencia de los pozos (de diversos sistemas y modos) que se atendían con los oficios disponibles en la estancia, la noria (que debía ser adquirida a un fabricante) requería otros conocimientos técnicos. ¿Cuáles serían las razones para invertir en esta herramienta? En primer término, la escala. Daireaux lo tiene muy claro:

Si la hacienda es mucha, una noria con malacate movido por un caballo, puede dar gran cantidad de agua en muy poco tiempo, y si todos estos medios son pocos para una hacienda considerable, queda la bomba de rosario que vierte mares, si el jahuel es abundante (1904: 79) [6].

El francés era terminante respecto de la importancia de garantizar el abastecimiento de aguas y convertirlo en una prioridad absoluta. Cuando se refería al pozo de balde/manga, lo describía como "sistema antiguo, primitivo, matador de caballos y de poco rinde" que debía ser reemplazado por el pozo semi-surgente. Y también afirma "El estanciero debe, lo mismo para las aguadas que para todo lo demás, calcular cuáles son sus recursos y sus necesidades, antes de adoptar tal o cual sistema; pero al tratar este renglón, debe ser liberal, generoso, con tino" (1904: 396).

Queda claro que había una dimensión de escala que hermanaba al pozo de balde con el jagüel y al molino con la represa australiana. A mayor volumen extraído, más importante debía ser el contenedor; obviamente esta variación iba a depender de las dimensiones del establecimiento y del plantel ganadero.

Veamos como analiza Hernández esta evolución:

Se ha usado en esta Provincia el balde sin fondo, la manga de lona ó de suela, y se usan actualmente diversas clases de baldes de fierro, equilibrados, de codo, con falso fondo y otros sistemas; pero el que consideramos mejor es el cajón de madera y manga de cuero, graduado y equilibrado, que puede en un momento y con la mayor facilidad, sacar una gran cantidad de agua. (1884: 137) 
Aún así, para grandes volúmenes el sistema preferido era el de la noria, incluso por consideraciones de tipo puramente económicas y de amortización de la inversión

lo mejor de todo es la noria para aquellos establecimientos de campo que tengan mucha hacienda, pues aunque cuesta más, emplea menos personal, menos caballos, dá mucha más agua y es de mucha duración. (1884: 134)

Quizás Daireaux fue quien más claro visualizó este proceso, cuando consideró al molino como la mejor solución tecnológica para este problema:

El pozo semi-surgente, con un molino de viento de gran tamaño, cuyas alas se mueven al menor soplo de viento, haciendo derramar continuamente un regular chorro de agua en una represa australiana de diez metros de diámetro, nos parece ser, por ahora el sistema más práctico para que la hacienda no llegue a carecer de agua. (1904: 80)

El molino de viento era una herramienta de máxima eficacia, que todavía cuenta con una presencia importante en los campos argentinos; reemplazó la tracción a sangre de los animales y preparó el contexto tecnológico para la incorporación del vapor. En su momento tuvo una rápida difusión, como podemos ver en el censo de 1895. Si en 1888 había 237 molinos "de viento" y la gran mayoría en la provincia de Buenos Aires, en 1895, se cuentan 939 nada más que en las provincias del litoral y 1811 máquinas "impulsadas por el agua ó utilizadas para elevarla (norias, malacates, ruedas hidráulicas, etc.)" (República Argentina, 1898: LVI)[7]

Incluso Zeballos nos llegó a presentar un escenario "mixto" en el cual había una gran red de aguas corrientes, con cañerías de fierro, por la cual circulaba agua extraída con un malacate de tracción a sangre

[...] Otro vasto galpón sirve para las máquinas de desgranar y moler maíz, romper cebada, pan de lino y picar pasto. Esta sección de máquinas se completa con una bomba de gran poder de extracción y elevación, para surtir de agua á todos los galpones y bebederos, hasta en los potreros, por medio de cañerías de fierro. El motor es un sólido malacate de caballos. (1888: 124)

Y finalmente, en la estancia "El molino" Zeballos podrá ver el máximo grado de desarrollo en gestión del agua para el período

Todo está servido por aguas corrientes, como también los galpones, huerta, patios, potreros y la casa habitación. El agua sale de un pozo de 15 varas de hondo, un molino á viento marca Corcoran del señor MIGUEL LANÚS y la eleva á un depósito de fierro á 10 varas de altura. La rueda tiene 12 pies de diámetro y con un par de horas de trabajo con buen viento, hay lo suficiente para todo el servicio y quedar llenos todos los depósitos. (1888: 108) 
Hay un párrafo que resulta muy esclarecedor respecto de la relación de los empresarios con relación a la gestión del agua

Si yo volviese á ser estanciero otra vez, dice el señor AMADEO, la primer cosa que haría, sería instalar un molino á viento: es todo cuanto puedo decir en su elogio. Ahora para no necesitar de peón ni siquiera para abrir las llaves que surten las bebidas en los potreros voy á poner, en vez de éstas, flotadores para que así que los animales beban, ella sola reponga el agua consumida. (1888: 108)

\section{Las aguadas naturales}

Se sabe que, durante muchos años, las aguadas funcionaron como las coordenadas para la adjudicación y mensura de los terrenos. Muchos documentos oficiales indicarían el límite frontal del terreno con la consabida fórmula "Tantas leguas de frente" sobre algún río e incluso sobre algún arroyo. También ofrecían soluciones productivas: brindaban agua natural para el consumo de los animales y contenían la hacienda, simplificando de alguna manera la labor de los puesteros para limitar los movimientos del ganado en los años previos al uso del alambrado. Como dice Hernández: "Ya hemos dicho que si el campo tiene rinconadas naturales formadas por rio ó arroyos, deben dejarse esas rinconadas para la hacienda, pues ahí bebe quieta, encuentra siempre pasto verde, se aquerencia y se acostumbra á respetar esa barrera" (1884, p. 168) y también Zeballos "Concurre sin duda á hacer inmejorable la calidad de este campo la existencia de dos arroyos permanentes de agua dulce que lo circunvalan casi completamente. Tales son el de "La Choza" y “El Durazno” (1888: 119).

El ferrocarril y la evolución de las técnicas de extracción de aguas subterráneas (que minimizaron la función hidrante de arroyos, cañadas y lagunas) hicieron mermar su importancia como valorizador de los terrenos. Hilando más fino veremos ventajas y desventajas.

En primer lugar (ya lo hemos visto) existía el riesgo de las inundaciones generados por los desbordes de los arroyos y lagunas. Si bien funcionan como drenajes de los terrenos altos, su situación los convierte en depositarias tarde o temprano de los volúmenes de agua que el suelo no pueda absorber. En esto la agencia activa del estanciero juega su carta fundamental para minimizar las dificultades. La "bendición” de un arroyo o una laguna requiere infraestructura, disposición logística y capacitación del personal, para convertirse en rentabilidad real.

Daireaux tiene especial consideración para este tema

Peligro gravísimo es el de dejar la majada, en estado de fatiga, tomar agua en arroyos correntosos; [...]

Conviene mucho desde el primer dia, marchar con los caballos adelante, cerca de la hacienda, pues le sirven de guia, el ganado se acostumbra á seguirlos, y esta guia es muy útil para los puentes, arroyos y pasos peligrosos. (162) 
En cuestiones sanitarias, al agua de las lagunas y arroyos se le debe destinar especial atención por diversas razones. El mal manejo de algunos escenarios atenta contra la rentabilidad, requiriendo por ello mucha atención de parte de los puesteros y mayordomos.

Diremos, de paso, que los cambios de agua enflaquecen el ganado.

Una hacienda que bebe á jagüel, y pasa de pronto á beber en las lagunas ó los arroyos, pierde gordura; y sucede también lo mismo si de las lagunas ó de los arroyos pasa al jagüel: el cambio de agua tiene el engorde en movimiento. (Hernández, 1884: 202)

En atención a estos detalles, el peón debe desarrollar conocimientos específicos respecto del comportamiento del organismo de los animales, pero también de la conducta de las ovejas ante diversos escenarios. Y como vemos, con escaso margen de error. Hernández se extiende al respecto:

Para pasar un rio es mejor que toda la hacienda venga mal bebida; - se apura para que pase y que beba en la orilla opuesta sino se hace asi, bebe, se dá vuelta y ya no pasa. (...)

En invierno cuando se derrita la helada, para el arreo, almuerza, después bebe, y sigue su marcha.

Cuando un arreo ha bebido debe marchar en seguida; este movimiento le hace bien después de tomar agua. $[\ldots]$

Si el conductor no es inteligente y precabido, y lleva su hacienda muy fatigada, donde quiera que se encuentra una laguna, los animales se lanzan á ella, beben, se opilan, se les enfria el sebo, y muere lo mas gordo. (Hernández, 1884: 322)

El inestable ciclo de lluvias de la región, con inviernos tan secos, hace que muchos de los cauces y lagunas terminen degenerando en "aguas muertas". Estas son una importante fuente de propagación de epizootias, por lo que se requiere tener alertas muy estrictas respecto de su conservación. Zeballos sugiere "Debe entonces alejarse constantemente el rebaño de las aguas muertas, de las cañadas [...] Las bebidas lavadas y restregadas á menudo con escobas del mismo pasto del campo" (1888: 281).

Y festeja la incorporación de nuevos conocimientos sobre las formas de preservar la salud de los animales

La noción divulgada de que las aguas de lagunas pequeñas, formadas por las lluvias, chapaleadas pronto por el ganado y trasformadas en lodazales, son el medio propicio para el desarrollo de gérmenes mortíferos, ha producido una trasformación saludable en los campos. (Zeballos, 1888: 253)

Hasta aquí hemos analizado la gestión del agua utilizada para beber, y también analizamos la importancia del control que debía ejercerse sobre el paisaje de la estancia, para evitar determinados fenómenos de acumulación de agua 
(anegamiento parcial o total) o de la existencia de aguas estancadas que propiciaran la existencia de bacterias nocivas para las ovejas. Porque, como decía Daireaux, la higiene que la oveja tenía como reglas generales la "Buena alimentación, más bien seca que acuosa, agua muy sana y pura y en muy pequeña cantidad buenos reparos contra la intemperie, holgura en el campo; con esto, y no existirá la caquexia" (312)[8].

\section{El agua en función sanitaria}

Para lograr una mejor calidad de lana, las estancias debían disponer grandes esfuerzos en la higiene de los animales. La dimensión sanitaria exigía grandes cantidades de agua, que se utilizaba tanto para la higiene propiamente dicha, como para la aplicación de productos medicinales. Hay una serie de medidas sanitarias destinadas a proteger el cuero y la lana, que insumen grandes cantidades de agua. El líquido no podía ser el mismo que el de los arroyos ya que -además- la aplicación se realizaba en galpones específicamente diseñados para ese uso.

Los animales enfermos de sarna producen menos lana y de menor calidad, afectando también a la calidad de los cueros. Para evitarla, las ovejas eran sometidas a baños de agua en las que se diluía algún elemento curativo. Zeballos comentaba que en aquellos lugares donde predominaba la "ganadería primitiva”, se seguían tradiciones indígenas como la grasa de jabalí o de potro. Despreciándolas, se inclinaba por soluciones de otra índole. Así, reseñaría una larga lista de productos que incluían glicerina, tabaco de Virginia, ácido carbólico, alquitrán con ácido fénico, etc. Algún productor le mencionaba (y esa era también la solución que Daireaux proponía) una combinación de azufre y cal viva. Todos estos productos debían diluirse en agua y en se aplicaba en forma manual, oveja por oveja o se sumergía al animal (durante cuatro o cinco minutos según Zeballos, un minuto según Daireaux) en agua especialmente preparada.

Para el tratamiento anti-sarna, se utilizaban las bañaderas, una pileta contenedora de líquido, que podía estar construida en madera impermeabilizada u otros materiales. Por allí pasaban las ovejas permaneciendo el tiempo estimado por el encargado de efectuar la labor. La dimensión de esta dependía del tamaño del establecimiento y la tecnología aplicada también. Zeballos menciona una estancia con un bañadero de piso de baldosas y con una leve inclinación del suelo, para que el agua que caía de las ovejas volviera a caer al bañadero para una nueva aplicación. En la estancia Plomer encontraría un equipamiento con un elevado standard tecnológico, donde las bombas de vapor son utilizadas para mover el agua ya utilizada

Bañadero. Este departamento tiene por objeto perseguir la sarna en todas las majadas, aprovechando la coyuntura oportuna del esquileo. Se compone de un primer cuerpo de máquinas para preparar y calentar el remedioá vapor y de un segundo cuerpo de máquinas para extraer el agua, con bomba también á vapor. El bañadero que sigue á estas construcciones, tiene 21 metros de largo y se complementa con tachos de fierro, 
brete, balanza que hace caer las ovejas al baño, corrales, trascorrales y galpón para depósito de combustible. (1888: 122)

Por su parte, Lemée, quien presta especial atención al tema sanitario, y describe el proceso realizado de una forma más arcaica y plantea un escenario en el cual las piletas se llenan con una bordalesa (225 litros) acarreada por dos hombres y afirma "Para bañar todo el dia sin con la pileta chica, se precisa un buen pozo, pero para bañar con la pileta grande, se precisan dos pozos, ó un pozo escepcional" (p. 63).

En la medida en que estas medidas están pensadas para mejorar la salud de los planteles, imaginamos que lograron seguir cumpliendo funciones durante la segunda fase de la producción ovejera en que el producto principal fue la carne de los animales.

\section{Reflexiones finales}

Siempre se ha hablado de las ventajas comparativas de la llanura pampeana con relación a otros lugares del mundo en la producción de bienes agropecuarios. De soslayo se menciona que la disponibilidad de agua no es parte menor de esa ventaja. Pero, una vez que un campo tenía agua (en cualquiera de sus fuentes) ¿qué se hacía con ella? Eso es algo a lo que los autores que hoy relevamos dan algunas respuestas con un nivel de detalle que nos permite mensurar la importancia del asunto. Los tres textos dejan en claro que, para crecer en escala y productividad, el recurso agua requería conocimientos técnicos y mejoras en la infraestructura, debido a que tanto la calidad del agua, el acceso y la posibilidad de controlar inundaciones fueron de vital importancia para el éxito de los emprendimientos.

Carmen Sesto ha puesto de relieve que la actitud innovadora de "la vanguardia ganadera" supo agregar a aquellas ventajas, inversiones en genética, infraestructura y sanidad (muchas de estas orientadas a la gestión del agua) para sumar rentabilidad. Creemos que una de las tareas para quienes aborden la historia del agua es la de reconstruir las conexiones entre el recurso y la producción, organizando descripciones sobre la forma en que determinadas estructuras sociales (en nuestro trabajo actual, las empresas agropecuarias en proceso de modernización) modelaron esas apropiaciones y en forma granular, una por una, fueron moldeando un espacio económico y productivo en la provincia de Buenos Aires.

Si bien las reseñas realizadas a los documentos están circunscriptas a una temática específica, consideramos que la información que organizamos permite ratificar algunas de las ideas que motivaron nuestro acercamiento al tema. Pudimos obtener algunas claves del rol del agua en la estancia ovejera, a partir del reconocimiento de su carácter híbrido (natural y social difícilmente disociado), integrando las perspectivas ambiental, económica y social, para sumar a una comprensión cabal de su importancia en el proceso económico agroexportador de fines del siglo XIX.

Vimos también que los modos de ocupación del espacio de estos establecimientos estaban muy lejos de ser pasivos respecto del agua; por el 
contrario, está claro que para garantizar el funcionamiento y la rentabilidad de esas estancias los empresarios debieron asignar recursos intelectuales y económicos para convertir esa ventaja comparativa que significaba la disponibilidad de agua en una posibilidad real de aprovechamiento. Los autores hoy mencionados, que eran además referentes políticos y del sector agropecuario, propusieron una guía de acción para la gestión y administración de las estancias y dejaron en claro el rol central que le cupo al agua como parte fundamental de ellas.

Quedarían por desarrollar investigaciones comparativas que incorporen la diversidad regional y si fuera posible, un trabajo "cruzado" con los inventarios de las estancias, para poder mensurar exactamente si los modelos planteados por estos autores fueron realmente implementados en los campos. Para ello, habría que ampliar el relevamiento de autores y sobre todo de casos de evidencia empírica respecto de los recursos que el agua consumía a los emprendimientos ganaderos.

El autor quiere consignar un agradecimiento muy grande (pero insuficiente) para el Dr. Juan Luis Martirén por sus indicaciones y orientaciones y también al Dr. Facundo Rojas, por sus aportes y señalamientos.

\section{Referencias citadas}

Amaral, S. (1998): The rise of capitalism on the pampas. The estancias of Buenos Aires. 1785-1870. Cambridge.

Bagaloni, V. (2018): “Desde los primeros puestos rurales hasta la estancia moderna del sur bonaerense: un recorrido arqueológico", Trabajos y Comunicaciones (47). doi: 10.24215/23468971e051

Balsa, J., y Colombo, G. (2007): "Estructura productiva y sujetos sociales en la expansión del ovino. El caso del partido de Saladillo en 1870", Mundo Agrario, 7(14). Recuperado de https://www.mundoagrario.unlp.edu. ar/article/view/v07n14a13

Bakker, K. (2012): "Water: Political, biopolitical, material”, Social Studies of Science 42(4), pp. 616-623.

Banzato, G. (2015): ““Convivir con el agua”: Las políticas de gestión del recurso hídrico ayer y hoy”, IV Jornadas Interdisciplinarias de Investigaciones Regionales, 7 al 9 de octubre de 2015, Mendoza, Argentina. Recuperado de http://www.memoria.fahce.unlp.edu. ar/trab_eventos/ev.4815/ev.4815.pdf

Banzato, G. (2016): Presupuestos y gastos del estado en la provincia de Buenos Aires para afrontar las inundaciones de los campos, 1870-1930. Revista Uruguaya de Historia Económica, 6 (9), 31-48. Recuperado de http://www.memoria.fahce.unlp.edu.ar/art_revistas/ pr.7436/pr.7436.pdf

Banzato, G. (2014): “Políticas públicas ante el cambio climático en un contexto de crecimiento económico: Construcción de una agencia estatal para paliar las inundaciones en la provincia de Buenos Aires, 1870 1910" [en línea], IV Congreso Latinoamericano de Historia Económica, 23 al 25 de julio de 2014, Bogotá, Colombia. Recuperado de http://www.memoria.fahce.unlp.edu. ar/trab_eventos/ev.3465/ev.3465.pdf

Barcos, M. y Martirén, J. (2019): “La metamorfosis de una economía agraria en la pampa argentina: Buenos 
Aires y Santa Fe entre las décadas de 1850 y 1890", Anuario de estudios americanos, 76 (2), pp. 585-614. Recuperado de http://www.memoria.fahce.unlp. edu.ar/art_revistas/pr.11130/pr.11130.pdf

Barsky, O. y Djenderedjian, J. (2003): Historia del capitalismo agrario pampeano. Tomo 1 La expansión ganadera hasta 1895, Buenos Aires, Siglo XXI.

Brittez, F (2006): "Instrucciones para estancieros" manuales de estancia y construcción del espacio pampeano en los albores del capitalismo industrial", Arqueología histórica en América Latina. Temasy discusiones recientes, compilado por P. Funari y F. Brittez, Mar del Plata, Ediciones Suárez.

Cortés Conde, R. (1998): “La economía de exportación argentina, 1880-1920”, Anuario IEHS 13.

Daireaux, G. (1904): La cría del ganado en la estancia moderna, Buenos Aires.

Djenderedjian, J. Bearzotti, S., Martirén, J. L. (2010): Historia del capitalismo agrario pamepeano: expansión agrícola y colonización en la segunda mitad del siglo XIX, Buenos, Aires, Siglo XXI

Flores, R. D. (2010): “Familias británicas en la sociedad rural”, épocas - revista de historia, 3, pp. 96-132.

Frid, C. (2007): “Del puerto al almacén rural: circuitos comerciales y producción lanar en el sur de Santa Fe (1860-1890)", Revista de Instituciones,

Ideas y Mercados, 46, pp. 93-116.

Gallo, Ezequiel (1983): La Pampa Gringa, Buenos Aires, Editorial Sudamericana.
Garavaglia, J. C. (1998): “Intensidad de uso de la tierra y tasas de ocupación ganadera en la pradera pampeana (1816-1852)", Quinto Sol, 2, pp. 5-23.

Garnero, G. (2014): "La dialéctica sociedad/cuenca hidrográfica en el valle de traslasierra. Las aguas del rio Mina Clavero, Córdoba 1870-1935”, Revista Pilquen-Sección Ciencias Sociales, 17 (2), pp. 1-16.

Garnero, G. (2014): Río Chico de Nono: una historia del vínculo sociedad y río en el oeste cordobés 18701935, Estudios Rurales.

Gelman, J. y Barsky, O., (2001): Historia del agro argentino, Desde la conquista hasta comienzos del siglo XXI, Buenos Aires, Siglo XXI.

Gómez Romero, F. (2016): “Investigaciones arqueológicas en el sitio ‘Estancia el Rosario’ del Juez Míguens, el primer editor del 'Martín Fierro' (Ayacucho, Buenos Aires)", Cuadernos de Antropología, 15, pp. 59-70.

Harratt, J. (1885): Estudios practicos sobre la cria y refinamiento del ganado lanar, Buenos Aires.

Hernández, J. (1884): Instrucción del estanciero, Buenos Aires, Editorial Casavalle.

Langhoff, M. L., Geraldi, A., Rosell, P. (2017): “El concepto de ciclo hidrosocial aplicado a los conflictos por el acceso al agua. El caso de la disputa por el río Atuel entre las provincias de La pampa y Mendoza, Argentina”, Papeles de Geografía, 63, pp. 146-160

Larsimont, R., \& Grosso, V. (2014): Aproximación a los nuevos conceptos híbridos para abordar las problemáticas hídricas”, Cardinalis, 2, pp. 27-48. Recuperado de 
https://revistas.unc.edu.ar/index.php/cardi/article/ view/7380

Lemée, C. (1882): Tratado de ganadería y agricultura escrito espresamente para la República Argentina con instrucciones muy detalladas de lo que le corresponde hacer al ganadero y al agricultor en cada mes del año, Buenos Aires, Coni.

Pérez Mendoza, D. (1863): Manual del pastor, Montevideo.

Posada, M. e Iuliano, R. (1995): “Modernidad y rentabilidad. La intelligentsia ganadera y los empresarios pecuarios: Godofredo Daireaux”, R.H.A., 119.

Provincia de Buenos Aires. (1870): Código Rural de la Provincia de Buenos Aires, ampliado con las modificaciones introducidas en el mismo por la Sociedad Rural Argentina, Buenos Aires, Imprenta Americana.

Rayes, A. (2014): “Sobreviviendo en el cambio. Las exportaciones argentinas de lanas y cueros en tiempos de cereales y frigoríficos, 1890-1913”, Quinto Sol, 18 (1).

Rayes, A. (2019): "Entre el crecimiento y la vulnerabilidad. Las exportaciones argentinas, c. 1875-1929”, Papeles de trabajo, 23(13).

Rayes, A. (2015): “Destinadas a un destino: Los inicios de las exportaciones argentinas de carnes frigoríficas, 1883-1913”, E.I.A.L., 26 (1).

Reguera, A. (1999): "Riesgo y saber: control y organización productiva en las estancias pampeanas (18801930)”, Anuario IEHS, 14 estancias pampeanas del sigloXIX. El caso de un gran empresario y propietario de tierras. Ciclos, AñoX, Vol. $\mathrm{X}, \mathrm{N}^{\circ} 20$, 2do semestre

Reguera, A. (1999): “Estancias pampeanas del siglo XIX. Estrategia empresaria para su funcionamiento: chacras agrícolas y puestos ganaderos", Quinto Sol, 3. pp.53-82.

República Argentina (1898): Segundo Censo de la República Argentina, Buenos Aires, Taller tipográfico de la penitenciaría nacional.

Sábato, H. (1989): Capitalismo y ganadería en Buenos Aires. La fiebre del lanar 1850-1890, Buenos Aires, Sudamericana.

Sbarra, N. (1973): Historia de las aguadas y el molino, Buenos Aires, Eudeba.

Santa Cruz, J. N. y Silva Busso, A. A. (2002): “Evolución hidrodinámica del agua subterránea en el conurbano de Buenos Aires, Argentina”, Boletín Geológico y Minero, 113 (3), pp. 259-272.

Schmit, R. (2015): “Estanciasy estancieros en Entre Ríos durante el siglo XIX, (1840 y 1880)", Mundo Agrario, 16 (31). Recuperado de http://www.mundoagrario. unlp.edu.ar/

Sempat Assadourian, C. (2011): "La economía del Río de la Plata durante el siglo XIX”, Anuario del Instituto de Historia Argentina, 11, pp. 11-26. Recuperado de: http://www.memoria.fahce.unlp.edu.ar/art_revistas/ pr.5241/pr.5241.pdf pag-15

Reguera, A. (2000): Estrategias de inversión en las 
Sesto, C. (2005): Historia del capitalismo agrario pampeano, Tomo II, La vanguardia ganadera bonaerense (1856-1900), Buenos Aires, Siglo XXI.

Sociedad Rural Argentina. (1873): Anales de la Sociedad Rural Argentina, Buenos Aires, Imprenta Americana.

Zeballos, E. (1888): A través de las cabañas, Buenos Aires, Peuser.

\section{Notas}

[1] El enteque seco se contrae cuando, ante la escasez de otras plantas, el ganado ingiere el "Duraznillo blanco" (Solanum glaucophyllum), una planta que crece en los bajos y que es altamente tóxica.

[2] Saguaypé o fasciola hepática es una parasitosis que ataca a bovinos, ovinos y porcinos. La enfermedad es considerada una de las parasitosis más importantes a nivel mundial origina disminución en la producción de carne, leche y lana. Los huevos del parásito son eliminados en las heces de los animales infestados. Una vez en el exterior su evolución se produce en pocos días y buscan un huésped intermediario, como el caracol de aguas dulces.

[3] Subrayado mío.

[4] Para un análisis contemporáneo y de larga duración respecto de las aguas subterráneas de la Provincia de Buenos Aires, ver Santa Cruz y Silva Busso (2002).
[7] SESTO trabajó con las cédulas censales que indican para 1895 una preeminencia de las norias sobre los molinos de viento en una proporción de 60 a 40, SESTO (114).

[8] Subrayado mío PS. 